\title{
Mechanisms of Respiratory Syncytial Virus Modulation of Airway Immune Responses
}

\author{
Matthew T. Lotz $\cdot$ R. Stokes Peebles Jr
}

Published online: 13 June 2012

(C) Springer Science+Business Media, LLC 2012

\begin{abstract}
Respiratory syncytial virus (RSV) most often causes severe respiratory disease in the very young and the elderly. Acute disease can also cause exacerbations of asthma in any age group. Recent findings provide insight into how the innate and adaptive immune systems respond to RSV infection and provide preliminary evidence that these effects vary significantly by RSV strain and host. Components of cell signaling pathways that induce inflammatory cytokine expression during the innate immune response and alter epithelial cell polarity through activating transcription factors, namely NF-kB, are now more clearly understood. New studies also reveal how RSV infection skews T helper (Th) cell differentiation away from the cell-mediated Th1 subset and towards the Th2 subset. There are also new data supporting preferential Th17 differentiation during RSV infection. In addition, effective immune system regulation of IL-10 expression and T regulatory cell (Treg) airway accumulation are essential for effective RSV clearance.
\end{abstract}

Keywords Respiratory syncytial virus (RSV) - T cells . Epithelial cells $\cdot$ Nuclear factor- $\mathrm{kB} \cdot \mathrm{NF}-\mathrm{kB} \cdot$ Inflammation $\cdot$ Modulation · Airway $\cdot$ Immune $\cdot$ Response $\cdot$ Asthma $\cdot$ Bronchiolitis

\section{T. Lotz $\cdot$ R. S. Peebles Jr}

Department of Medicine, Division of Allergy,

Pulmonary and Critical Care Medicine,

Vanderbilt University School of Medicine,

Nashville, TN, USA

R. S. Peebles Jr $(\bowtie)$

T-1217 Medical Center North,

Vanderbilt University Medical Center,

116121 st Avenue South,

Nashville, TN 37232-2650, USA

e-mail: stokes.peebles@vanderbilt.edu

\section{Introduction}

Respiratory syncytial virus (RSV) is an enveloped negative sense single-strand RNA virus of the Paramyxoviridae family and pneumovirus genus. RSV infection is most common in infants and young children: nearly all children are infected by 2 years of age [1]. RSV infection of infants can lead to serious respiratory disease, sometimes requiring hospitalization. In a screen for viral and atypical bacterial respiratory pathogens, RSV was the most prevalent pathogen $(43.3 \%)$ infecting children less than 5 years old with acute respiratory infection (ARI), as well as extremely common agent $(44.1 \%)$ in co-infection cases of 2 or more pathogens in the same cohort [2].

In conjunction, it has long been speculated that acute RSV infection during infancy correlates with a greater risk of allergic asthma later in life $[3,4]$. This correlation is evident in a longitudinal study by Sigurs and colleagues of 47 children who were hospitalized with RSV lower respiratory tract infection (LRTI) when they were less than 1 year old [5••]. In a recent follow-up with the patients at age 18, the RSV LRTI cohort had increased incidence of asthma with recurrent wheeze, asthma without recurrent wheeze, and allergic rhinoconjunctivitis (ARC) compared with 92 age-matched control patients (Table 1) [5••]. In addition, the LRTI cohort had increased sensitization to animal dander and perennial allergens as well as decreased spirometric function [5••].

These and other epidemiological data support 2 notions. RSV could have a long-lasting effect via an alteration of the immune response; it is also possible that early RSV infection serves as an indication of inherent differences in an individual's immune system, which leaves it not only vulnerable to serious RSV infection, but also to other, chronic respiratory conditions (eg, recurrent wheeze and asthma). It is also possible that these hypotheses work in combination. 
Table 1 Allergic symptoms in 18-year-old cohort

\begin{tabular}{lll}
\hline & $\begin{array}{l}\text { RSV } \\
\text { group } \\
(\mathrm{n}=46)\end{array}$ & $\begin{array}{l}\text { Control } \\
\text { group } \\
(\mathrm{n}=92)\end{array}$ \\
\hline Current asthma/RW** & $39 \%$ & $9 \%$ \\
Current asthma only** & $33 \%$ & $7 \%$ \\
$\begin{array}{l}\text { Allergic rhinoconjunctivitis (ARC)* } \\
\text { Atopic dermatitis }\end{array}$ & $43 \%$ & $17 \%$ \\
$\begin{array}{c}\text { Animal dander sensitization* } \\
\text { (Positive skin prick test) }\end{array}$ & $11 \%$ & $9 \%$ \\
$\begin{array}{c}\text { Perennial allergen sensitization** } \\
\text { (Positive skin prick test) }\end{array}$ & $33 \%$ & $11 \%$ \\
$\begin{array}{c}\text { Positive Phadiatop response* (Allergic } \\
\text { sensitization as defined by Phadiatop) }\end{array}$ & $56 \%$ & $14 \%$ \\
\hline
\end{tabular}

* $P \leq 0.005 ; * * P \leq 0.001$

Many of the RSV studies have explored these broad hypotheses by examining the effects of RSV on different aspects of the immune response in both human patients and mouse models. Recent work has done a great deal to expound the mechanisms underlying the immune response to RSV infection through investigating the formative signaling pathways and genetic underpinnings to the subsequent differential cellular responses. RSV infection causes a wide array of immunologic responses; clarifying the ways in which RSV triggers these changes may lead to the development of effective therapeutics that can ameliorate or abolish acute and chronic disease that result from ineffective immune system response.

\section{Initial RSV Infection, Cell Signaling, and the Innate Immune System Response}

RSV predominantly infects primary airway epithelial cells, but can also infect other structural airway and immune cells. The host cells recognize RSV via toll-like receptors (TLRs). RSV commonly activates TLR2, TLR3, and TLR4 [6-9]. In addition, new research shows TLR7 activation during RSV infection [10•]. These receptors in turn promote expression and secretion of inflammatory cytokines (Table 2), which mount the earlier innate immune response and subsequent adaptive immune response. To elicit these responses, several different signaling pathways and their respective molecules are utilized. These include pathways that include protein kinase $\mathrm{C}$ (PKC), mitogen-activated protein kinase (MAPK), and nuclear factor- $\mathrm{kB}(\mathrm{NF}-\mathrm{\kappa} \mathrm{B})$.

p38 MAPK and extracellular signal-regulated kinase (ERK) MAPK are both involved in RSV replication in human airway epithelial cells [11]. Pharmacologic inhibitors for p38 and ERK (SB203580 and U0126, respectively) both decreased viral replication when cells were treated 30 minutes prior to infection. In primary fibroblasts from myeloid differentiation factor 88 (MyD88) knockout (KO) mice, RSV-induced p38 activation was MyD88-dependent, whereas ERK activation was MyD88-independent. In the presence of anti-TLR4 antibodies, p38 MAPK activation decreased, whereas ERK MAPK remained unchanged. In addition, immunofluorescence confocal microscopy revealed that TLR4 clustering at the point of contact between the virus and cell was related to p38 activation, helping to elucidate the mechanism of viral entry [11].

Upon viral entry and activation of signaling complexes, inflammatory cytokines and chemokines are expressed and secreted. One important chemokine during this response is IL-8, which, as a chemoattractant, recruits neutrophils to the location of infection [12]. RSV induces IL-8 expression, which is mediated by NF- $\mathrm{BB}$ and AP-1 [13]. In RSVinfected A549 cells, co-transfection of a reporter luciferase gene coupled to the IL- 8 promoter, and a dominant negative (DN) mutation revealed that either of the 2 IKK $\beta$ DNs used significantly decreased IL- 8 promoter activation. An IKK $\beta$ inhibitor, NEMO binding domain (NBD) fusion peptide, significantly decreased IL-8 secretion. Therefore, NF-kB was activated through the canonical pathway. In addition, RSV infection increased JNK kinase activity. Inhibition of JNK significantly reduced transfected AP-1 reporter activity, while leaving NF- $\kappa$ B reporter activity unaffected [14••].

The regulation of JNK and IKK $\beta$ was also examined. MEKK1 and TAK1 are serine/threonine kinases that activate both JNK and IKKs [15, 16]. When A549 cells were infected with RSV, TAK1 was essential to both AP-1 and NF- $\kappa B$ activation [14••]. Thus, RSV infection caused IL8 expression through NF- $\mathrm{KB}$ and AP-1 induction via the

Table 2 Summary of cytokines and chemokines produced during RSV infection

\begin{tabular}{lll}
\hline Cytokine & Source & Effects \\
\hline IFN- $\gamma$ & NK cells, CD4 ${ }^{+}$T cells, CD8 ${ }^{+}$T cells & Increases NK activation, Th1 differentiation \\
IL-1 $\beta$ & Macrophages & Increases expression of cytokines mediated by NF- $\mathrm{BB}$ \\
IL-4 & CD4+ T cells & Increases Th2 differentiation, decreases Th1 differentiation \\
IL-8 & Epithelial cells, Macrophages & Neutrophil recruitment \\
IL-10 & CD4+ T cells & Inhibits expression of inflammatory cytokines, suppresses inflammatory response \\
IL-17A & CD4+ T cells & Increases Th17 differentiation, increases inflammatory cytokine production
\end{tabular}


canonical and the JNK pathway, respectively, and TAK1 was an upstream regulator able to mediate both responses. However, TAK1 depletion did not entirely abrogate NF- $\mathrm{KB}$ activation and nuclear translocation. Thus, there are likely other kinases that regulate IKK and NF-KB activation during RSV infection [14••].

The canonical pathway is not the only recently reported means of induction of NF- $\mathrm{KB}$ during RSV infection in human airway epithelial cells. In addition, the importance of a non-canonical pathway mediated by the virus-activated kinase (VAK) complex (composed of TBK-1 and IKK $\varepsilon$ ) is also evident [17]. In RSV-infected 293 cells transfected with IKK $\varepsilon$ DN, IL-8 promoter activity was significantly downregulated in comparison to control cells at 12 hours postinfection. Using siRNA in A549 cells and mouse embryonic fibroblasts from IKK $\varepsilon$ KO mice, IKK $\varepsilon$ was determined to regulate NF- $\mathrm{kB}$ during RSV infection through p65 Ser536 phosphorylation [17]. Thus, there is evidence for 2 separate pathways of NF- $\mathrm{kB}$ activation during RSV infection.

In a similar vein, RSV infection leads to IL- $1 \beta$ expression, which is an inflammatory cytokine critical to antiviral immune response. IL-1 $\beta$ works in an autocrine and paracrine fashion, and ultimately causes the expression of a number of NF-kB-dependent cytokines and chemokines [18]. Mature IL-1 $\beta$ is produced and secreted by activated macrophages following assembly and activation of inflammasomes in the cytoplasm $[19,20 \bullet]$. This is achieved through 2 signals: first, stimulation of a pattern recognition receptor ensures expression of pro-IL-1 $\beta$ (the IL- $1 \beta$ precursor) and inflammasome components; second, the inflammasome complex assembly and caspase- 1 activation cleave pro-IL- $1 \beta$ into IL- $1 \beta$. This second signal is either initiated by reactive oxygen species (ROS), cellular potassium efflux, or cathepsin leakage into the cytosol after lysomal disintegration. NF- $\mathrm{KB}$ activation through a TLR2/MyD88-dependent pathway is necessary for the first signal of IL- $1 \beta$ production in macrophages during RSV infection. The second signal is a combination of ROS production and potassium efflux via an ATP-sensitive potassium channel [20•]. These new findings elucidate the basic mechanism of IL-1 $\beta$ production in RSV-infected macrophages.

Likewise, RSV induces IL-15 expression in both epithelial cell lines and primary bronchial epithelial cells. IL-15 is produced by the canonical pathway induction of NF-KB [21]. IL-15 is an inflammatory cytokine that is suggested to augment antiviral defense through upregulation of IFN-related transcription factors, NF- $\mathrm{kB}$, synthesis of type 1 effector molecules, and survival and stimulation of $\mathrm{NK}$ cells and $\mathrm{CD} 8^{+} \mathrm{T}$ cells [22-24]. Thus, effective expression of IL-15 could be beneficial to effective RSV clearance; further research is required to determine the importance of IL-15 in shaping the immune response to RSV infection.
In addition to regulating inflammatory cytokine production, NF- $\mathrm{KB}$ activation is associated with tight junction formation in RSV-infected human nasal epithelial cells. Tight junctions are important for epithelial cell polarity and maintenance of tissue integrity. During RSV infection, genes for tight junction molecules, claudin-2, $-4,-7,-9,-14$, -19; occludin; ZO-2; cingulin and MAG-1 were upregulated at least 2 -fold. NF- $\mathrm{KB}$ signaling through $\mathrm{PKC} \delta$ up-regulated claudin-4 expression [25]. Interestingly, inhibition of NF- $\mathrm{KB}$ and $\mathrm{PKC} \delta$ led to decreased viral replication and decreased formation of virus filaments. This, along with immunocytochemistry of tight junction proteins and viral proteins (RSV proteins $F$ and $G$ ) seem to suggest that virally-induced tight junction formation could augment cell polarity during RSV infection allowing for characteristic viral budding at the apical surface [25].

\section{Adaptive Immune System Response}

RSV infection also leads to a specific adaptive immune response. The characteristics of the adaptive immune response (ie, which $\mathrm{T}$ helper cell subset and associated cytokines dominate the response [Table 2]) can help to understand acute RSV disease and the implication of RSV infection for potential longterm respiratory disease. For instance, allergic asthma is characterized by a Th2-mediated response [26]. Therefore, it is important to realize any effects RSV infection has on predisposition to a certain adaptive immune response and the mechanism by which it occurs. RSV infection has been associated with skewing the immune system away from an anti-viral Th1 response and towards a Th 2 response. New data shed light on how this occurs and introduced a role of IL-17A (the principle cytokine of Th17 response) in RSV infection as well, as antibody blockade of IL-17A led to significant reduction of viral protein mRNA expression in the lungs and Th2 cytokine protein expression in the lymph nodes in RSV-infected mice compared with mice treated with control antibody [27]. This is interesting because IL-17A has recently been implicated in the development of severe forms of asthma [28].

Epithelial cells, in addition to providing a link to the initial innate immune response, aid in adaptive immune system activation after RSV infection. When human bronchial epithelial cells (HBECs) were co-cultured with peripheral blood mononuclear cells (PBMCs), there was an induction of IFN- $\gamma$, IL-4, and IL-17 cytokine production, which suggested activation of lymphocytes as these are the characteristic cytokines of T helper cell subsets Th1, Th2, and Th17, respectively [29]. When isolated lymphocytes were exposed to the supernatant of RSV-infected HBECs, flow cytometry revealed that compared with controls exposed to complete cell culture medium, Th2 and Th17 differentiation was significantly induced, while Treg differentiation was clearly suppressed [29]. In addition to 
inherent major histocompatibility complex (MHC) I, epithelial cells may be able to express MHC II in times of stress or disease, as well as costimulatory molecules CD80 and CD86 during infection [30-32]. Therefore, it is conceivable that HBECs directly activate naïve $\mathrm{CD}^{+}$lymphocytes during RSV infection.

Epithelial cells could also activate lymphocytes by an indirect means. RSV infection of primary rat airway epithelial cells (PRAECs) caused upregulated thymic stromal lymphopoietin (TSLP) 6 to 18 hours post-infection [33]. When RSV-infected PRAECs were cocultured with myeloid dendritic cells (mDCs), the mDCs had increased major histocompatibility complex II (MHC II) and CD86, but not CD80 expression. In the same coculture system, siRNA for TSLP effectively decreased TSLP expression and also the expression of MHC II and CD86, without changing CD80 expression. In this $\mathrm{mDC}$ - and RSV-infected PRAEC coculture system, mDCs also up-regulated mRNA expression of molecules important for Th2 polarization: thymus- and activation-regulation chemokine (TARC) and OX40L, while TNF- $\alpha$ mRNA was down-regulated. Functional mDC maturation was verified, as mDCs exposed to RSV-infected PRAECs significantly increased $T$ cell proliferation in comparison to uninfected PRAECs [33]. Thus, it is possible that RSV infection of epithelial cells leads to a differential $\mathrm{T}$ helper cell response through the polarization of $\mathrm{mDCs}$, and potentially directly through epithelial cell antigen presentation. However, this study did not assess the ability of affected mDCs to alter naïve T cell differentiation. Further study is necessary to further elucidate the mechanisms of $\mathrm{T}$ cell activation and differentiation during RSV infection.

During RSV infection, natural killer (NK) cells may also have a role in $\mathrm{T}$ cell differentiation [34]. Interestingly, past clinical studies show that increased NK deficiency is linked to increased respiratory disease symptoms. Specifically, NK cell numbers were reduced in hospitalized bronchiolitis cases, and ventilated infants exhibited an even greater (3-fold) reduction in NK cells. In fatal RSV bronchiolitis cases, patients were nearly devoid of NK cells [35-37]. In BALB/c mice, antibody-mediated NK cell reduction during RSV infection resulted in lower IFN- $\gamma$ production and an increase in Th2 cytokines. This result was dependent on IL-25 produced by airway epithelial cells: IL-25 up-regulated Jagged1 on dendritic cells and led to increased Th2 differentiation [34].

\section{Immune System Regulation During RSV Infection}

Just as the immune system needs to effectively activate and clear a pathogen, it also needs an effective system of selfregulation and inhibition to inhibit an unbalanced immune response and collateral damage of host tissue. Studies examining humans and mouse models reveal that inefficient immune system regulation escalates respiratory disease pathology following RSV infection, and can also lead to less optimal viral clearance.

IL-10 is an extremely important regulatory cytokine. It mediates suppression of the activation and effector functions of many immune system cells. As can be expected, it is important in RSV infection and resultant disease pathogenesis. In hospitalized infants with RSV bronchiolitis, IL-10 concentration in nasopharyngeal aspirates were significantly higher in infants who later developed post-bronchiolitis wheeze (PBW) than in those who did not [38]. However, this difference in IL-10 concentration was not correlated to the rs1800872 single-nucleotide polymorphism (SNP) in the IL10 promoter region. This SNP was considered because heterozygous expression was previously correlated with protection from RSV bronchiolitis [39, 40]. So, although there is some support for a protective role from this IL10 genetic variation, the potential functional role during RSV infection remains unclear [38].

That said, it is also possible that during RSV infection the IL-10 response is modulated via indirect genetic factors. For instance, IL-19 and IL-20 are both IL-10 family cytokines and their genes are clustered with IL10 (as well as IL24) [41-43]. In 1 recent study of 166 hospitalized infants with RSV LRTI, 1 SNPs of the IL19 gene (rs2243191 and rs2243188) and 1 SNP of IL20 gene (rs2981573) were associated with protection from recurrent wheeze (OR 0.4, 0.5 , and 0.4 , respectively). From these SNPs, 3 haplotypes comprised $99 \%$ of all the IL19/IL20 haplotypes in the patients. Only 1 of these haplotypes, TGG (the nucleotides of rs2243191, rs2981572, and rs2981573, respectively), was significantly related to recurrent wheeze after LTRI. It had a protective role, with an odds ratio of 0.43 [44]. Perhaps changes in IL-10 family cytokine expression through SNPs play a role in direct immune system regulation during RSV infection. It is also possible that their expression influences IL-10 expression during infection because IL-10 family members cross-regulate each other in other models [44, 45].

These cohort studies show that IL-10 has some influence in disease pathogenesis, prompting studies to focus on elucidating the mechanism of IL-10 and other factors of immunosuppression during RSV infection in mouse models. $\mathrm{CD}^{+}$and $\mathrm{CD}^{+} \mathrm{T}$ cells both produced IL-10 in the lung during RSV infection in mice, with production peaking at 8 days post infection [46•]. The majority of IL-10 producing T cells were $\mathrm{CD}^{+}$during RSV infection. The vast majority of $\mathrm{CD}^{+} \mathrm{T}$ cells that produce IL-10 co-produced IFN- $\gamma$, while this was less true for $\mathrm{CD}^{+} \mathrm{T}$ cells [47]. However, $\mathrm{CD}^{+}$cells also may have modulated IL-10 production, as depletion of $\mathrm{CD}^{+}$cells led to a significant increase in IL-10. Thus, these data support that $\mathrm{CD}^{+}$cells play a suppressive role in $\mathrm{CD}^{+}{ }^{\mathrm{IL}}-10$ production [46•]. 
Although $\mathrm{CD}^{+}{ }^{+} \mathrm{T}$ cells are the major producers of IL-10 in mouse RSV infection, the subset that contributes most to this production is not as clear. One group has shown $\mathrm{T}$ regulatory cells $\left(\mathrm{CD} 4^{+} \mathrm{Foxp}^{+}\right)$was the most common IL10 expressing cells in the lung, mediastinal lymph node (MLN) and BAL fluid of infected mice, while another has shown that $\mathrm{CD}^{+}{ }^{+} \mathrm{Foxp}^{-}$are the majority of IL-10 expressers $[46 \cdot, 47]$. Thus, it needs to be determined under which circumstances, if any, each subset expresses the most IL-10.

In IL-10 KO mice and WT mice treated with anti-IL-10R antibody, RSV infection increased disease and delayed recovery when compared to IL-10 producing and control antibody treated mice, respectively [46•, 47]. IL-10 depletion was associated with increased weight loss, airway obstruction, edema, tissue inflammation, and mucus production. These macroscopic changes were marked by a congruous increase in inflammatory cytokines (IL-6, IL17A, IL-17F, IFN-y, TNF-a) and chemokines (CXCL1, CXCL10, CXCL9, CCL1, CCL2, CCL3) [46•, 47-49]. Thus, a lack of IL-10 resulted in an altered adaptive immune response, providing evidence that IL-10 is an important regulator of inflammation during RSV infection. This is reflected at the cellular level: with IL-10 depletion there was a decrease in total cell recruitment to the BAL at day 4, whereas there was increased airway inflammation 6 and 8 days post-infection $[47,49]$. This trend could be responsible for the lag in viral clearance in comparison to control mice. In addition, there was a decrease in natural $\mathrm{T}$ regulatory cells $\left(\mathrm{CD}^{+}{ }^{+} \mathrm{Foxp}^{+}{ }^{+} \mathrm{Helios}^{+}\right)$when mice were given anti-IL-10R antibody [46•].

As IL-10 is an important regulatory cytokine, $\mathrm{T}$ regulatory cells (Tregs; $\mathrm{CD}^{+}{ }^{+} \mathrm{Foxp}^{+}$) are responsible for suppressing the adaptive immune response. They are, therefore, instrumental to effective host response to RSV infection. After RSV infection in mice, there was a rapid accumulation of Tregs in the airways and secondary lymphoid tissue. By 4 days postinfection there is a 50-fold increase in Tregs in the BAL fluid of infected mice compared to naïve mice. This drastic increase was amplified 8 days post-infection to an 86-fold increase over naïve mice. There was also significant Treg accumulation observed in the lung parenchyma and MLNs and a slight increase in the spleen [50].

Local proliferation of Tregs occurred with $78 \%$ and $69 \%$ of Tregs expressing the proliferation marker Ki-67 in the BAL and lung parenchyma, respectively, 6 days post-infection [50]. In addition, the majority of Tregs expressed activation markers, which were up-regulated post-infection. $\mathrm{CD} 43^{\text {glyco }}$, ICOS, CTLA-4, CD69, OX40, and PDL-1 were all significantly increased 6 days post-infection. $\mathrm{CD} 25^{+}$Tregs present in the airways increased significantly as well. With the clearance of RSV, the number of Tregs decreased, returning to baseline numbers. Treg efflux took longer in the BAL and MLNs than in the lung parenchyma and spleen [50].
Tregs are important in the immune response to RSV infection. A $60 \%$ decrease in $\mathrm{CD} 4{ }^{+} \mathrm{Foxp}^{+} \mathrm{CD} 25^{+}$Tregs using CD25-neutralizing antibodies was extremely detrimental to immune system efficiency, leading to a significant increase in time of viral clearance. This delay could be traced to a delay in the recruitment in RSV-specific $\mathrm{CD} 8^{+}$ $\mathrm{T}$ cells to the lung [50]. This finding argues that Tregs are more than just immunosuppressors; they also act as conductors of an effective immune response for RSV clearance.

\section{Different Strains, Different Cells: Different Response}

As can be surmised in understanding the vast and intricate effects of RSV infection on the immune system response, it is important to acknowledge that there is variability of immune response to RSV infection from case to case. There are many different strains of RSV that each contains significant genetic variability, leading to altered virulence and subsequent disease pathogenesis. Also, the characteristics of the infected host cell (ie, species and type of cell) can lead to differential cellular and systematic responses. This is especially important to keep in mind in examining in vitro studies that focus on a small number of cell types. Obviously, it is necessary to use basic systems to garner mechanistic understanding, but we must be mindful of the plasticity inherent in these mechanisms and their phenotypic manifestations. Inherent differences lead to differences in the innate and adaptive immune responses triggered by RSV infection.

Several recently published studies examine the effects of intrinsic differences on RSV pathogenesis and immune response. In examination of the pathogenesis of several RSV isolates in a BALB/cJ mouse model, both laboratory strains (A2, Long, and line19) and clinical strains (A2001/2-20, A2001/3-12, A1997/12-35, A2000/3-4, A1998/3-2, and A1998/12-21) were studied [51•]. Of the laboratory strains, only A2 caused host weight loss, while 3 of 6 clinical isolates (3-12, 2-20, 12-35) caused weight loss post-infection. While those 4 isolates caused weight loss, they all caused different patterns of weight loss: early, late, and bimodal patterns were all represented. Similarly, strains caused differing characteristics and disease phenotypes including: varying expression of lung IL-13 and gob-5 protein, amount of in vitro replication, in vivo viral load, and other characteristics. Fundamental differences in immune response followed these variations in the observable effects of disease. Indeed, in a comparison of A2, 2-20, 3-12, and line 19 at 8 days post-infection, A2 infection had a significantly greater amount of $\mathrm{CD} 8^{+} \mathrm{T}$ cells in the lung that produced IFN- $\gamma$ than 2-20 and line 19 . Interestingly, this matched PAS staining data that showed A2 as nonmucogenic, whereas $2-20$ and line 19 were mucogenic. This finding links the role of $\mathrm{T}$ cell differentiation and disease pathogenesis [51•]. 
A similar study examined a variety of RSV strains (A2 and 3 clinical isolates: BT2a, BT3a, BT4a) in primary human bronchial epithelial cells (PBECs) from healthy children [52]. As in the aforementioned mouse model, the strains were characterized by different patterns of infection. The strains differed drastically in their ability to infect PBECs, as revealed by host cell expression of viral F protein. A2 was much more infectious; at 72 hours post-infection, most host cells were infected. In contrast, the clinical isolates were not nearly as infectious, and displayed differential infectivity (BT2a was most infectious, BT4a was least). This difference carried over to viral growth kinetics: A2 grew more quickly and to higher titers than the clinical isolates. A2 titers reached $5.25 \log _{10}$ $\mathrm{TCID}_{50} / \mathrm{mL}$ at an MOI 0.1, whereas the clinical isolates BT2a, BT3a, and BT4a reached 4.20, 3.80, $3.25 \log _{10} \mathrm{TCID}_{50} / \mathrm{mL}$, respectively. Interestingly, all strains initiated a post-infection increase in the concentration of the chemokines and cytokines RANTES, IP-10, IL-6, and IL-8 in supernatants. However, by 72 hours post-infection, A2 had significantly higher concentrations of IP-10 and RANTES than the mock infections and clinical isolates. Similarly, IL-8 was significantly up-regulated by only A2 and BT4a and IL-6 only by BT4a [52]. So, inflammatory chemokine and cytokine release varied by RSV strain, which would likely elicit a variable immune system response in an in vivo model.

From the opposite perspective, the types of cells used for experimental infection are also important. For instance, a group examined RSV infection in airway epithelial cells (AECs) from lungs of healthy children and cells of the BEAS-2B cell line [53•]. They determined that the peak of RSV replication (as determined by real-time PCR for RSV Ngene expression) was 2 to $3 \log$-fold greater in AEC cultures than in BEAS-2B cultures. Similarly, cytotoxicity was greater in AECs. Although both cell culture supernatants contained high concentrations $(>500 \mathrm{pg} / \mathrm{mL})$ of IL- 8 and IL- 6 from 3 to 48 hours post-infection, AECs had significantly greater concentrations of IL-8 than BEAS-2B from 6 to 24 hours postinfection [53•]. In short, the response to RSV infection differed between epithelial cells derived from epithelial cell lines and from the lungs of children.

\section{Conclusions}

Recent advancements in RSV research have illuminated the signal transduction pathways that are activated to initiate transcription of inflammatory cytokines and chemokines once RSV is recognized by a TLR on the cell surface. In addition, Th2 and Th17 differentiation and role in disease progression during RSV infection are better understood. That said, it is necessary to better characterize the link between the innate and adaptive immune systems post-infection, and how variations in this link can lead to variations in acute and chronic airway disease. Understanding the influence of regulatory agents (eg, IL-10, Tregs) host genetic predisposition, viral strain, and other factors on this combined immune response may help glean new therapeutic avenues.

Acknowledgment This work was funded in part by National Institutes of Health grants R01 HL 090664, R01 AI 070672, R01 AI 059108, GM 015431, R21 HL106446, and U19AI095227, and a Veteran Affairs grant (1I01BX000624).

Disclosure No potential conflicts of interest relevant to this article were reported.

\section{References}

Papers of particular interest, published recently, have been highlighted as:

- Of importance

•. Of major importance

1. Glezen WP, Taber LH, Frank AL, Kasel JA. Risk of primary infection and reinfection with respiratory syncytial virus. Am J Dis Child. 1986;140:543-6.

2. Bezerra PG, Britto MC, Correia JB, et al. Viral and atypical bacterial detection in acute respiratory infection in children under five years. PLoS One. 2011;6:e18928.

3. Frick OL, German DF, Mills J. Development of allergy in children. I. Association with virus infections. J Allergy Clin Immunol. 1979;63:228-41.

4. Pullan CR, Hey EN. Wheezing, asthma, and pulmonary dysfunction 10 years after infection with respiratory syncytial virus in infancy. Br Med J (Clin Res Ed). 1982;284:1665-9.

5. •- Sigurs N, Aljassim F, Kjellman B, et al. Asthma and allergy patterns over 18 years after severe RSV bronchiolitis in the first year of life. Thorax. 2010;65:1045-52. A longitudinal study that examines the correlative relationship between early RSV infection and later allergy and asthma development.

6. Haynes LM, Moore DD, Kurt-Jones EA, et al. Involvement of tolllike receptor 4 in innate immunity to respiratory syncytial virus. J Virol. 2001;75:10730-7.

7. Groskreutz DJ, Monick MM, Powers LS, et al. Respiratory syncytial virus induces TLR3 protein and protein kinase R, leading to increased double-stranded RNA responsiveness in airway epithelial cells. J Immunol. 2006;176:1733-40.

8. Kurt-Jones EA, Popova L, Kwinn L, et al. Pattern recognition receptors TLR4 and CD14 mediate response to respiratory syncytial virus. Nat Immunol. 2000;1:398-401.

9. Murawski MR, Bowen GN, Cerny AM, et al. Respiratory syncytial virus activates innate immunity through Toll-like receptor 2. J Virol. 2009;83:1492-500.

10. • Lukacs NW, Smit JJ, Mukherjee S, et al. Respiratory virus-induced TLR7 activation controls IL-17-associated increased mucus via IL23 regulation. J Immunol. 2010;185:2231-9. Role of TLR 7 in host recognition of $R S V$ and subsequent immune response.

11. Marchant D, Singhera GK, Utokaparch S, et al. Toll-like receptor 4-mediated activation of p38 mitogen-activated protein kinase is a determinant of respiratory virus entry and tropism. J Virol. 2010;84:11359-73.

12. Remick DG. Interleukin-8. Crit Care Med. 2005;33:S466-7.

13. Garofalo R, Sabry M, Jamaluddin M, et al. Transcriptional activation of the interleukin- 8 gene by respiratory syncytial virus 
infection in alveolar epithelial cells: nuclear translocation of the RelA transcription factor as a mechanism producing airway mucosal inflammation. J Virol. 1996;70:8773-81.

14. •• Dey N, Liu T, Garofalo RP, Casola A. TAK1 regulates NFKappaB and AP-1 activation in airway epithelial cells following RSV infection. Virology. 2011;418:93-101. This study shows that in RSVinfection of A549 cells, NF-kB is activated by the canonical pathway and that AP-1 is JNK-dependent. Both of these pathways are regulated by TAK1. This elucidates the signaling pathway that results in $I L-8$ expression during RSV infection.

15. Ninomiya-Tsuji J, Kishimoto K, Hiyama A, et al. The kinase TAK1 can activate the NIK-I kappaB as well as the MAP kinase cascade in the IL-1 signalling pathway. Nature. 1999;398:252-6.

16. Zhou L, Tan A, Iasvovskaia S, et al. Ras and mitogen-activated protein kinase kinase kinase-1 coregulate activator protein-1- and nuclear factor-kappaB-mediated gene expression in airway epithelial cells. Am J Respir Cell Mol Biol. 2003;28:762-9.

17. Bao X, Indukuri H, Liu T, et al. IKKepsilon modulates RSVinduced NF-kappaB-dependent gene transcription. Virology. 2010;408:224-31.

18. Weber A, Wasiliew P, Kracht M. Interleukin-1 (IL-1) pathway. Sci Signal. 2010;3: $\mathrm{cm} 1$.

19. Davis BK, Wen H, Ting JP. The inflammasome NLRs in immunity, inflammation, and associated diseases. Annu Rev Immunol. 2011;29:707-35.

20. - Segovia J, Sabbah A, Mgbemena V, et al. TLR2/MyD88/NFkappaB pathway, reactive oxygen species, potassium efflux activates NLRP3/ASC inflammasome during respiratory syncytial virus infection. PLoS One. 2012;7:e29695. Helps elucidate the mechanism of $I L-1 \beta$ production in macrophages during RSV infection.

21. Zdrenghea MT, Telcian AG, Laza-Stanca V, et al. RSV infection modulates IL-15 production and MICA levels in respiratory epithelial cells. Eur Respir J. 2012;39:712-20.

22. Liu K, Catalfamo M, Li Y, et al. IL-15 mimics T cell receptor crosslinking in the induction of cellular proliferation, gene expression, and cytotoxicity in CD8+ memory T cells. Proc Natl Acad Sci U S A. 2002;99:6192-7.

23. Foong YY, Jans DA, Rolph MS, et al. Interleukin-15 mediates potent antiviral responses via an interferon-dependent mechanism. Virology. 2009;393:228-37.

24. Freeman CM, Han MK, Martinez FJ, et al. Cytotoxic potential of lung CD8(+) T cells increases with chronic obstructive pulmonary disease severity and with in vitro stimulation by IL-18 or IL-15. J Immunol. 2010;184:6504-13.

25. Masaki T, Kojima T, Okabayashi T, et al. A nuclear factor-kappaB signaling pathway via protein kinase $\mathrm{C}$ delta regulates replication of respiratory syncytial virus in polarized normal human nasal epithelial cells. Mol Biol Cell. 2011;22:2144-56.

26. Bosniak B, Stelzmueller B, Erb KJ. Epstein MM Treatment of allergic asthma: modulation of Th2 cells and their responses. Respir Res. 2011;12:114.

27. Mukherjee S, Lindell DM, Berlin AA, et al. IL-17-induced pulmonary pathogenesis during respiratory viral infection and exacerbation of allergic disease. Am J Pathol. 2011;179:248-58.

28. Wang YH, Wills-Karp M. The potential role of interleukin-17 in severe asthma. Curr Allergy Asthma Rep. 2011;11:388-94.

29. Qin L, Hu CP, Feng JT, Xia Q. Activation of lymphocytes induced by bronchial epithelial cells with prolonged RSV infection. PLoS One. 2011;6:e27113.

30. Kalb TH, Chuang MT, Marom Z, Mayer L. Evidence for accessory cell function by class II MHC antigen-expressing airway epithelial cells. Am J Respir Cell Mol Biol. 1991;4:320-9.

31. Kurosawa S, Myers AC, Chen L, et al. Expression of the costimulatory molecule B7-H2 (inducible costimulator ligand) by human airway epithelial cells. Am J Respir Cell Mol Biol. 2003;28:563-73.
32. Papi A, Stanciu LA, Papadopoulos NG, et al. Rhinovirus infection induces major histocompatibility complex class I and costimulatory molecule upregulation on respiratory epithelial cells. J Infect Dis. 2000;181:1780-4.

33. Qiao J, Li A, Jin X. TSLP from RSV-stimulated rat airway epithelial cells activates myeloid dendritic cells. Immunol Cell Biol. 2011;89:231-8

34. Kaiko GE, Phipps S, Angkasekwinai P, et al. NK cell deficiency predisposes to viral-induced Th2-type allergic inflammation via epithelial-derived IL-25. J Immunol. 2010;185:4681-90.

35. De Weerd W, Twilhaar WN, Kimpen JL. T cell subset analysis in peripheral blood of children with RSV bronchiolitis. Scand J Infect Dis. 1998;30:77-80.

36. Welliver TP, Garofalo RP, Hosakote Y, et al. Severe human lower respiratory tract illness caused by respiratory syncytial virus and influenza virus is characterized by the absence of pulmonary cytotoxic lymphocyte responses. J Infect Dis. 2007;195:1126-36.

37. Welliver TP, Reed JL, Welliver Sr RC. Respiratory syncytial virus and influenza virus infections: observations from tissues of fatal infant cases. Pediatr Infect Dis J. 2008;27:S92-6.

38. Schuurhof A, Janssen R, de Groot H, et al. Local interleukin-10 production during respiratory syncytial virus bronchiolitis is associated with post-bronchiolitis wheeze. Respir Res. 2011;12:1-7.

39. Hoebee B, Bont L, Rietveld E, et al. Influence of promoter variants of interleukin-10, interleukin-9, and tumor necrosis factor-alpha genes on respiratory syncytial virus bronchiolitis. J Infect Dis. 2004; 189:239-47.

40. Janssen R, Bont L, Siezen CL, et al. Genetic susceptibility to respiratory syncytial virus bronchiolitis is predominantly associated with innate immune genes. J Infect Dis. 2007;196:826-34.

41. Blumberg $\mathrm{H}$, Conklin $\mathrm{D}, \mathrm{Xu} \mathrm{WF}$, et al. Interleukin 20: discovery, receptor identification, and role in epidermal function. Cell. 2001;104:9-19

42. Gallagher G, Dickensheets H, Eskdale J, et al. Cloning, expression and initial characterization of interleukin-19 (IL-19), a novel homologue of human interleukin-10 (IL-10). Genes Immun. 2000;1:442-50.

43. Dumoutier L, Leemans C, Lejeune D, et al. Cutting edge: STAT activation by IL-19, IL-20 and mda-7 through IL-20 receptor complexes of two types. J Immunol. 2001;167:3545-9.

44. Ermers MJ, Janssen R, Onland-Moret NC, et al. IL10 family member genes IL19 and IL20 are associated with recurrent wheeze after respiratory syncytial virus bronchiolitis. Pediatr Res. 2011;70:518-23.

45. Jordan WJ, Eskdale J, Boniotto M, et al. Human IL-19 regulates immunity through auto-induction of IL-19 and production of IL10. Eur J Immunol. 2005;35:1576-82.

46. - Weiss KA, Christiaansen AF, Fulton RB, et al. Multiple CD4+ T cell subsets produce immunomodulatory IL-10 during respiratory syncytial virus infection. J Immunol. 2011;187:3145-54. Explores the origin and importance of IL10 expression during RSV infection.

47. Loebbermann J, Thornton H, Durant L, et al. Regulatory T cells expressing granzyme B play a critical role in controlling lung inflammation during acute viral infection. Mucosal Immunol. 2012;5:161-72

48. Tsuchida T, Matsuse H, Fukahori S, et al. Effect of respiratory syncytial virus infection on plasmacytoid dendritic cell regulation of allergic airway inflammation. Int Arch Allergy Immunol. 2012;157:21-30.

49. Newcomb DC, Boswell MG, Huckabee MM, et al. IL-13 regulates Th17 secretion of IL-17A in an IL-10-dependent manner. J Immunol. 2012;188:1027-35.

50. Fulton RB, Meyerholz DK, Varga SM. Foxp3+ CD4 regulatory T cells limit pulmonary immunopathology by modulating the CD8 T cell response during respiratory syncytial virus infection. J Immunol. 2010;185:2382-92. 
51. - Stokes KL, Chi MH, Sakamoto K, et al. Differential pathogenesis of respiratory syncytial virus clinical isolates in BALB/c mice. $\mathrm{J}$ Virol. 2011;85:5782-93. Evidence that different RSV strains impact the development of airway disease and immune system response differently in mice.

52. Villenave R, O'Donoghue D, Thavagnanam S, et al. Differential cytopathogenesis of respiratory syncytial virus prototypic and clinical isolates in primary pediatric bronchial epithelial cells. Virol J. 2011;8:43.

53. - Fonceca AM, Flanagan BF, Trinick R, et al. Primary airway epithelial cultures from children are highly permissive to respiratory syncytial virus infection. Thorax. 2012;67:42-8. Evidence that airway epithelial cells derived from cell lines have different responses to RSV infection from primary epithelial cells. 